“Transfer” XV: 1-2 (2020), pp. 276-294. ISSN: 1886-554

\title{
EL UNIVERSO EDUCATIVO DE IDA BACCINI: PASIÓN, COMPROMISO Y PERSONALIDAD
}

Salvatore Bartolotta (ORCID: 0000-0002-2386-5408) -

María Angélica Giordano Paredes (ORCID: 0000-0003-4519-6572)

Universidad Nacional de Educación a Distancia (UNED)

El siglo XIX marca una nueva época de luchas y reivindicaciones políticas, sociales y de género. La voz femenina se hace eco a través de la literatura autobiográfica, ya en voga en autores reconocidos, y como forma de "autorrepresentación" fuera del canon preestablecido, que como bien se sabe, estaba representado por los-hombres. Pero es a finales de ese siglo que la palabra de la mujer adquiere una considerable connotación social y literaria en una nación que acababa de redefinir su destino (Covato 2006). Estos hechos se llevaron a cabo en un ámbito renovado, con un curioso espíritu patriótico, en el que la moral y el ejemplo constituían el eje principal de una cultura que pretendía ser mejor, a través del buen comportamiento civil y religioso. A pesar de ser un país laico, de amplia representación burguesa, el peso del catolicismo fue destructor de ideales, entre ellos la igualdad de derechos, aun a pesar de grandes controversias con la nueva moral laica, nacida de los ideales republicanos..

Ida Baccini, como otras mujeres de la época, comprometidas con sus ideales educativos y progresistas, entretejió su vida con la labor didáctica e intelectual que llevó a cabo en múltiples facetas, empezando por la experiencia docente, que promulgó en su autobiografía, poniendo en evidencia su gran personalidad y su fehaciente decisión en desacuerdo con la legislación vigente. No titubeó al momento de decidir el final de su carrera de maestra ya que, para ella, era más importante la libertad de sus ideas que la sumisión al yugo de la imposición de una ley educativa de la que estaba totalmente en contra. 
“Transfer” XV: 1-2 (2020), pp. 276-294. ISSN: 1886-554

\begin{abstract}
M'ero licenziata dalla scuola elementare alla fine del 1878 ed eccone le ragioni. Alla piccineria dell'ambiente e alle frequenti sciocchezze di un cattivo metodo pedagogico che urtava profondamente i miei sistemi d'idee -idee tutte giovani, sane ed essenzialmente moderne-, s'era aggiunta in quell'anno una prescrizione municipale che finì col farmi saltare la mosca al naso: l'obbligo della ginnastica. Non approvavo il provvedimento per molte ragioni: alcune di indole puramente pedagogica, altre di indole esclusivamente femminile. (Baccini 1904: 143)
\end{abstract}

De esta manera dejó la actividad docente para emprender una nueva etapa más productiva en la que dio rienda suelta a su talento para innovar el sistema educativo italiano. El nuevo camino editorial le permitió publicar libros educativos, ejemplares y moralizantes, para no ir en contra de los preceptos de la época y hacer llegar a los jóvenes, principalmente a las mujeres, un mensaje de reconstrucción y de modernidad, de cambio social y de reivindicación silenciosa poniendo en discusión los roles tradicionales en el ámbito familiar. Sin embargo, y a pesar de su rebeldía y anticonformismo con el sistema vigente, le reconocieron los méritos en un certificado que merece la pena detallar a continuación y que ella incluyó en su autobiografía:

[...] la signora Ida Baccini, nominata, in seguito a concorso per esame, maestra assistente nelle Scuole elementari comunali il 22 gennaio 1873 , vi ha disimpegnato il proprio ufficio con zelo ed operosità veramente esemplari, fino a tutto il giorno presente. Attesta altresì che la predetta signora Ida Baccini, dotata di non comune ingegno, dimostrò coltura di gran lunga superiore a quella richiesta per occupare il posto che Le fu conferito e che nelle Scuole sovraindicate tenne sempre un contegno lodevolissimo, così per cortese deferenza verso i suoi superiori, come per gentile amorevolezza verso gli alunni che le furono affidati, dalla disciplina e dal profitto dei quali si ebbero prove non dubbie della sua attitudine all'insegnamento elementare [...]. (Baccini 1904: 145)

El certificado es un ejemplo del patriotismo de la época; pero llaman la atención, de manera particular, los halagos a la inteligencia de Ida Baccini "dotata di non comune ingegno, dimostrò 
“Transfer” XV: 1-2 (2020), pp. 276-294. ISSN: 1886-554

coltura di gran lunga superiore a quella richiesta [...]" Una mujer de inteligencia superior a la media y de grandes capacidades que había demostrado ser excelente en su trabajo. Esos halagos tienen una clara explicación en el mismo contexto histórico de la época. Las mujeres, por su condición de madres y por su evidente sensibilidad, eran las mejores candidatas para educar a los niños tanto dentro como fuera del ámbito doméstico. Es por eso que durante la unidad de Italia ellas tuvieron un rol muy importante en la formación de la juventud que constituiría el futuro glorioso de una gran nación forjada en los ideales de Mazzini entre otros héroes del resurgimiento italiano. Ida, sin embargo, fue muy crítica con el sistema:

Ed ora due parole sui sistemi pedagogici di quel tempo (1871-78) applicati nelle scuole elementari del Comune di Firenze. Non desiderando che questi miei appunti biografici dieno argomento a pettegolezzi e, peggio ancora, a malignità, tacerò il nome della scuola e degl'insegnanti alla cui ombra io feci il mio tirocinio di maestra [...] Mutai altre scuole, ma i sistemi, su per giù erano quelli: le classi, un accasermamento di poveri ragazzi pigiati sui banchi come le sardine in una botte: gl'insegnanti tanti poveri pastori scontenti, armati di un bastone per tener nelle file il gregge. (Baccini 1904: 111-114)

Estas palabras de la escritora reflejan su gran inquietud por los avances, por una educación que diera respuestas a las curiosidades de los discentes, que los hiciera partícipes de la construcción de su propia historia, con espíritu crítico y moderno y con una actitud pragmática frente a sus propias necesidades. De hecho, no dudó en criticar tanto la actitud del sistema como el comportamiento de los protagonistas del mismo, "pastores" y "ovejas", obligados a usar la fuerza para mantener la disciplina y transmitir los conocimientos. Profesores dotados de sabiduría y alumnos pasivos, asustadizos y domados por un bastón que imponía la autoridad del más fuerte.

Por todas estas razones, de sobrada importancia, dejó la enseñanza y comenzó una nueva aventura como escritora y periodista, sin dejar de lado el campo pedagógico, al que le dedicó el 
“Transfer” XV: 1-2 (2020), pp. 276-294. ISSN: 1886-554

resto de su vida en cada uno de sus libros y de sus métodos de enseñanza y aprendizaje (Cini 2013). Cada cuento que escribió estaba cargado de una clara intención educativa; por lo que se puede afirmar que fue siempre fiel a su compromiso didáctico, intentando hacer fuera de la escuela y por medio de las palabras, lo que no consiguió en su carrera docente de muy corta duración. "Il suo interesse nasce dallo studio dei bambini e delle giovani donne che si dedicano all'insegnamento. Le maestre avranno il sacro ufficio di seminare nei giovani cuori e nelle avide intelligenze dei fanciulli nobili sentimenti e alti pensieri" (Cini 2013: 108).

Para ella la profesión docente debe ser, necesariamente, vocacional porque es una gran responsabilidad forjar el futuro de una persona y el de una nación, por consiguiente. Con esta convicción trazó el recorrido de su vida profesional, volcada siempre en los jóvenes. Empezó trabajando en revistas y periódicos como la Gazzetta d'Italia y la Rivista Europea, utilizando el nombre de su hijo como pseudónimo, Manfredo, para evitar que le obstaculizaran su camino y poder así expresar sus opiniones sobre lo que debía ser un buen educador y un buen sistema educativo. De hecho, sus libros son un ejemplo de buena didáctica y de implicación en la formación de los jóvenes italianos de la época.

En 1895 empezó a publicar en el Giornale dei Bambini', "nel quale il tono palesemente moraleggiante è mitigato da un modello romantico di educazione. All'interno di questa rivoluzione culturale si afferma la stampa periodica per l'infanzia" (Cini 2013: 112). Páginas enteramente llenas de mensajes educativos de altísimo alcance moral que tenían por finalidad formar una mentalidad sana, sabia y respetuosa de los derechos y deberes de los ciudadanos. Ida Baccini se supo ganar su espacio y sus obras fueron conocidas en la época, especialmente, Memorie di un pulcino; pero, lamentablemente, no tuvieron trascendencia y con el paso de los años cayeron en el olvido, sustituidas por obras de igual, o incluso menor importancia, pero de autores masculinos que fueron los que pasaron a la historia. Por lo tanto, pese a su esfuerzo y enco-

${ }^{1}$ El 7 de julio de 1881 se publicó en Roma el primer número del Giornale per i Bambini, la primera revista semanal dedicada exclusivamente a los niños. 
“Transfer” XV: 1-2 (2020), pp. 276-294. ISSN: 1886-554

miable labor, la obra de Ida Baccini ya no hace eco en nuestra sociedad.

Sin embargo, en su ardua labor periodística encontró el espacio necesario para dar voz a sus inquietudes, que se articulará en torno a la formación de la juventud que constituirían el futuro de Italia, y que tanto le preocupaban. En esa época de cambios y de exigencias culturales, se atrevió a tomar la iniciativa y a seguir la corriente de esa sociedad que necesitaba modernizarse y canalizar mejor sus ideales (Soldani 2004). Era necesario adecuarse a los nuevos tiempos y, sobre todo, al modo de encauzar la didáctica; pero el recorrido que empezaba a emprender no correspondía a sus convicciones pedagógicas, por lo que decidió luchar por cambiar ese rumbo a través de la escritura. De allí su convincente rol editorial a través de la creación de manuales escolares con una metodología bien razonada e intencionada y una literatura dirigida a la formación intelectual, espiritual y moral de los jóvenes. Gracias al nuevo "status" de los intelectuales en la reciente unificación nacional y a la exigencia de una mejor escolarización, muchas mujeres tuvieron la oportunidad de acceder a la industria cultural y producir literatura, ejerciendo de escritoras, conferenciantes, profesoras, traductoras, educadoras, cronistas, maestras, ensayistas, redactoras de periódicos y directoras de revistas (D'Agostino 2009), entre ellas Ida Baccini de cuya obra se hablará detenidamente, ya que se dedicó a la elaboración de manuales escolares y de comportamiento, memorias, artículos periodísticos, novelas y cuentos, en lo que se empieza a considerar una literatura consumista y contribuyó con su escritura

all'unificazione linguistica, culturale e sociale, mentre l'industria editoriale e libraia traeva degli eccezionali benefici dallo sviluppo della produzione letteraria femminile, che mostrava una straordinaria capacità comunicativa con il pubblico di lettori. (D'Agostino 2009: 188) 
“Transfer” XV: 1-2 (2020), pp. 276-294. ISSN: 1886-554

De hecho, Pietro Dazzi, ${ }^{2}$ en el prólogo de Lezioni e racconti per i bambini y, dirigiéndose al editor Trevisini, alude a la obra de la escritora, bastante conocida en la época, como:

libri di testo [...] che vengono letti molto volentieri dai nostri fanciulli e sono adoperati in moltissime scuole italiane. Dettati nel puro e gentile idioma toscano, questi libri parlano al fanciullo il suo linguaggio, lo dilettano, lo avvezzano a vedere, ad osservare il mondo esteriore, come ad amare il bene morale. Prima di accingersi a scrivere libri scolastici, l'Autrice si fece maestra, e nelle scuole, in mezzo a centinaia di fanciulli, imparò a conoscerne il cuore, e vide il modo di mettere in pratica i principi della scienza pedagogica moderna. Perciò i libri della signora Baccini, scritti con esperienza della scuola e con cuore pieno di carità, sono veri giojelli per l'infanzia. Sia dunque certo, signor Trevisini, che questo libriccino riceverà liete ed oneste accoglienze, perchè la signora Baccini scrive con pratica di insegnante e con cuore di artista e di madre. Tale è la mia opinione espressa così alla buona a Lei; ma se Ella credesse che queste quattro righe possano stare in fronte al nuovo libretto, le stampi pure. (Baccini 1882: 6)

E Ida Baccini responde al escritor y académico defendiendo su espíritu moderno y su gran compromiso con la educación:

Fra i libri scolastici elementari, apparsi in quest'ultimo biennio, molti sono senza dubbio quelli che si raccomandano agli educatori, sì per la bontà degli intendimenti, come per la forma snella e schiettamente italiana [...] Di libri però che istruendo educhino, e che nell'analisi dei sentimenti osservino quella eterna e quasi sempre dimenticata legge di gradazione, senza l'osservanza della quale cresceremo alla società non degli uomini, ma delle caricature, pare a me sia tuttora difetto grande. Tutti i nostri fanciulli, che pur cianciano eruditamente della semplicità di Cincinnato e

\footnotetext{
${ }^{2}$ Lo que sabemos de Pietro Dazzi (1838-1896) lo enlaza, en cierto modo, con Ida Baccini en cuanto a intelectual consagrado y comprometido con la Italia unida, académico de la Crusca y educador. Es más conocido por su labor de fundador de las escuelas profesionales que estaban destinadas a la preparación cultural y técnica de las masas populares.
} 
“Transfer” XV: 1-2 (2020), pp. 276-294. ISSN: 1886-554

della grandezza del primo Bruto, si danno i pizzicotti tra loro, fanno le boccacce al maestro e mettono in canzonatura la nonna. Facciamoci piccoli coi piccoli, e se non riusciremo a fabbricar degli omìni, avremo pur sempre il gusto di vederci crescer d'intorno dei ragazzi buoni e garbati [...] Con questi intendimenti mi sono ingegnata di mettere insieme il mio nuovo libriccino. Ė un'umile cosa: ma se la lettura di queste paginette potesse far buono un fanciullo, o se cattivo, correggerlo, a me parrebbe di avere inalzato una piramide. (Baccini 1882: 7)

En esta humilde confesión de la escritora se aprecia, claramente, la intención educativa y moral de su obra, de cada una de las historias de vida familiar, de la intrahistoria italiana del resurgimiento que no es más que la preocupación por formar hombres y mujeres educados, cultos y capaces de hacer de Italia una nación grande, rica y respetada. Esos eran unos de los ideales de ese periodo histórico que supo conjugar la experiencia con los preceptos religiosos y morales para crear un modelo de sociedad. Prueba de ello son los relatos que exaltan a Eduvige (Una donnina), la niña que pese a su edad se encargó de las labores domésticas para ayudar a su padre que tenía que salir de casa a trabajar; ya que la madre estaba en la cama enferma. Un modelo heroico de mujercita que se dedica al hogar y a los demás, altruista y generosa, la mujer pasiva que está condenada a las paredes domésticas; pero que representa, al mismo tiempo, un instante de reflexión sobre la condición de la mujer en el siglo XIX. No hay que olvidar que Ida Baccini utilizó el silencio y la resilencia para luchar contra la sumisión de la mujer, entretejiendo historias reales, a veces estrechamente ceñidas a las reglas morales de la sociedad, pero necesarias para hacer reflexionar a las jovencitas que las leían y se instruían en un ambiente de buen comportamiento, o incluso exagerado sentido del deber. De hecho, el relato de Eduvige, como los de todos los héroes urbanos y rurales a los que la escritora se refiere, terminan con una moraleja cuya intención, además de educar, es, claramente, la de criticar silenciosamente ese modelo de sociedad "C'è una gran soddisfazione a studiare e a meritarsi il premio: ma quella di rendersi utile alla mamma malata è più gran- 
“Transfer” XV: 1-2 (2020), pp. 276-294. ISSN: 1886-554

de di tutte!" (Baccini 1882: 10). Si bien es cierto que es virtuoso cultivar la bondad y ayudar a los demás; es menos cierto y criticable que una niña de nueve años renuncie a su infancia para hacer las labores de casa y ayudar a su padre, justificando, en cierto modo, el machismo y el rol patriarcal de la figura masculina; y por supuesto, la desigualdad de género.

Enrichetta (Il fratellino dell'Enrichetta) recibe una lección de maternidad cuando el padre le hace entender el rol de la mujer durante la lactancia y la crianza de los hijos, descuidándose a sí misma para dedicarse, de lleno, a la educación de los niños "La mamma rinunziò per te a ogni divertimento, a ogni spasso: trascurò tutte le sue conoscenze e si dette esclusivamente a te: quando, a volte, era costretta a uscir di casa per far delle compre, stava sempre in pensiero e non vedeva l'ora d'esser tornata" (Baccini 1882: 18). El relato hace reflexionar sobre la función biológica de la mujer que la somete a los cuidados del menor renunciando a la libertad de tomar decisiones fuera de ese ámbito doméstico. Por un lado está bien que la mujer se dedique a sus hijos y los eduque con amor y sabiduría; pero por otra parte, no es admisible tal sometimiento a la naturaleza y a la servidumbre doméstica. Las lecciones morales de Ida Baccini tienen una doble faz con la misma clara intención didáctica, la de ser buenas personas, en este caso mujeres, y la de escrudiñar a fondo cada situación y encontrar el mensaje de la revolución muda.

La literatura italiana está llena de mujeres que representan los mismos cánones femeninos, símbolos de belleza, abnegación y sometimiento; o de maldad y destrucción.

La donna, nata, cresciuta ed educata solo per essere moglie e madre esemplare e alla quale sono stati affidati per millenni tutti i compiti relativi alla gestione della casa e dell'educazione della prole, almeno nell'infanzia, misteriosamente scompare quando si tratta di citare criteri come saggezza, giustizia, rigore. Il messaggio risulta chiaro: le donne devono agire, ma in subordine e non ambire ad alcun merito che, di riflesso spetta al capofamiglia. Ma è proprio così? Le donne sono delle eterne minori, irrazionali, incapaci di autodeterminare la propria esistenza, le proprie scelte, 
“Transfer” XV: 1-2 (2020), pp. 276-294. ISSN: 1886-554

in sintesi la propria vita? O è necessario un punto di vista diverso, un approccio differente rispetto a quello di Dante o in seguito di Cattaneo, di De Sanctis o di Croce nell'affrontare il discorso sull'esistenza e sull'agire delle donne nella storia e nella cultura italiana? (Moschini 2013: 14)

Este modelo, creado a conveniencia de la cultura patriarcal que no quería perder el predominio en las decisiones de la vida conyugal y de la comunidad, tiene sus primeros ecos de desobediencia en los personajes de Ida Baccini, que irradiaban sabiduría y justicia, pese al sometimiento tradicional al que estaban condicionados; ya que como ella misma decía, eran "caricaturas" de esa sociedad. A eso contribuía la educación y justo era el arma con el que ella se habría propuesto luchar contra el sistema patriacal. Así, en Lavori e balocchi (Il primo lavoro della Gemma), la escritora nos describe a una niña que quiere impresionar a su madre con un regalo y utiliza todo su ingenio e inteligencia para idearlo; sin dejar de lado la reflexión, teñida de sabiduría y humildad, al pedir consejos a otra mujer, con más experiencia y conocimientos, como la maestra. En este caso ambos personajes demuestran que son capaces de pensar y de utilizar la inteligencia para tomar una decisión o, simplemente para elegir la mejor opción, la más conveniente y sencilla, pero que consiguiera emocionar a la madre.

La Gemma aveva messo da parte nientemeno che settantacinque centesimi; e con settantacinque centesimi si possono fare dimolte cose: si può comprare una bambola quasi vestita, un anellino quasi d'oro, un vezzo di perle quasi buone, o se no, un mezzo chilogrammo di biscottini. Ma la Gemma aveva un'altra idea: voleva fare un regalo alla mamma e non sapeva proprio dove battersi il capo. Pensa e ripensa, si decise di confidarsi con la sua maestra. E questo è il partito più savio al quale possa appigliarsi una bambina imbarazzata. La Gemma avrebbe voluto comprar tutto Firenze, e se tutto Firenze non c'entrava, si sarebbe contentata di una bella pelliccia di martora, col manicotto eguale. Ma la signora maestra che era una signorina di giudizio, fece osservare alla fanciulla, che con settantacinque centesimi non si compra neanche la fodera del manicotto. 
“Transfer” XV: 1-2 (2020), pp. 276-294. ISSN: 1886-554

- O allora? - fece la Gemma sgomenta. - Capirà, signorina, che io non posso regalare alla mamma una trombettina o una palla di gomma elastica! (Baccini 1882: 58)

La frase, "la signora maestra che era una signorina di giudizio" (Baccini 1882: 58), muestra un ejemplo de personaje femenino dotado de razonamiento y agudeza mental. De esta manera se puede ver a la mujer tradicional que se ocupa de los quehaceres domésticos pero que es capaz de ser autónoma como ser pensante y racional sin depender de la voluntad de nadie. La mujer, en este caso, viene tratada al mismo nivel del hombre, en igualdad de virtudes y capacidades. Es verdad que es una mujer rural y doméstica; pero que da un paso importante en esa sociedad que estaba empezando a cambiar. La autora, sin embargo, no deja de implantar la huella didáctica porque para Ida Baccini cualquier situación es válida para enseñar a los niños a ser mejores personas y a actuar con parsimonia y sentido común, aunque vaya siempre cargada de ejemplaridad y parezca, a veces, excesivamente artificial. La maestra, además de aconsejar a Gemma, la convence para que haga algo económico y creativo, demostrando así tanto el ingenio como el razonamiento bien calculado para contribuir a la economía doméstica. Es decir, el modelo de persona que razona y utiliza su inteligencia para el bien de su familia; pero que, aunque es una niña, podría ser también un hombre, solo que Ida Baccini quiere utilizar estos elementos para transmitir ese mensaje silencioso que es, a la vez, una clase de comportamiento social y de ética donde la presencia femenina es un aliciente para despertar la consciencia de las mujeres y hacerles entender que no hay roles diferenciados sino actuaciones y comportamientos positivos, eficaces, para el buen funcionamiento de la sociedad. Por eso sus relatos son rotundamente educativos y cada uno de ellos está considerado como un modelo pedagógico para enseñar a los futuros ciudadanos italianos a ser mejores personas para poder construir una gran nación. No cabe duda de que el patriotismo está presente en cada personaje y en cada historia y que cada personalidad está influida por el peso de la religión, que junto a la moral, constituían el eje de la sociedad; pero también está claro 
“Transfer” XV: 1-2 (2020), pp. 276-294. ISSN: 1886-554

que ser buenas personas depende de la formación, la buena educación y el desarrollo de las capacidades cognitivas que eran la prioridad de la pedagogía y de la existencia de escritoras y maestras como Ida Baccini.

Los relatos de Lezioni e racconti per i bambini eran verdaderos manuales con los que pretendía enseñar la lengua y las demás asignaturas básicas en la escuela italiana de la época como geografía e historia, ciencias naturales, manualidades, etc. Se puede apreciar la intención de la escritora en la contextualización de los contenidos, ya que no se trataba de una metodología técnica y estática sino de su aplicación mediante ejemplos reales que transmitían los comportamientos de los personajes, en lo que hoy denominamos competencias pragmática y sociocultural. De esta manera el aprendizaje era más dinámico e interactivo y creaba más emoción en los niños e ilusión por un aprendizaje con inminente finalidad práctica, aplicada a las necesidades de la vida real. Ida Baccini se alejaba así del método abstracto que se imponía en las escuelas de la época y que hoy en día se sigue utilizando, después de dos siglos de evolución o involución de la sociedad. Por todo esto se puede afirmar que Ida Baccini fue una innovadora y una mujer muy moderna y avanzada para la época y que su legado no merece ser almacenado en el olvido sino reconsiderado y aplicado.

Relatos como Per un chicco di grano que utiliza para explicar como cultivar la tierra y el valor de la agricultura:

- Mi sapresti dire che cosa fa quell'uomo?

- Quell'uomo, figliuolo mio, mette a profitto la forza dei suoi bovi, i quali, come vedi, si tirano dietro l'aratro, per arare la terra e disporla alla sementa del grano. Sai già che l'aratro è lo strumento più importante dell'agricoltura e serve a tracciare nel terreno $i$ solchi profondi che dovranno accogliere il nuovo seme. (Baccini 1882: 21)

A través del diálogo transmite conocimientos lingüísticos y culturales como el léxico específico (l'aratro, arare, grano, seme, etc.) y las funciones lingüísticas estrechamente relacionadas con 
“Transfer” XV: 1-2 (2020), pp. 276-294. ISSN: 1886-554

los referentes culturales que son el cultivo de la tierra como medio de sustento, además de valores sociales y éticos, como la importancia del trabajo en la vida de una persona.

Con Fuoco e fiammiferi elabora una verdadera unidad didáctica de química, interactiva y práctica, mediante la cual facilita la asimilación de esos contenidos científicos tan difíciles para los niños, y que, a través de un cuento no sólo son más fáciles de adquirir sino más motivadores, cautivando así, la atención de los niños:

In Amburgo, che è una città della Germania, viveva un certo Brandt, mercante di condizione. Pare che la mala riuscita dei suoi affari lo persuadesse a cercare una via di guadagno nelle ricerche dell'Alchimia, parola con la quale gli uomini di cui vi ho parlato battezzavano i loro ridicoli tentativi. Standosene un giorno nel suo laboratorio, intento a far bollire al fuoco violento d'un gran fornello diverse sostanze, fra le quali era mescolata dell'orina, ottenne inaspettatamente e con suo grandissimo stupore, non l'oro agognato, non il rimedio universale, ma una materia singolare, strana, somigliantissima alla cera bianca. Aveva un leggiero odore d'aglio e, cosa più bizzarra ancora, risplendeva nell'oscurità. Quest'ultima proprietà le valse il nome di fosforo, che vuol dire portaluce.

Le molteplici esperienze fatte da altri uomini dotti provarono che questa sostanza esiste in gran quantità nelle ossa di tutti i mammiferi cioè di quegli animali che nascono colle forme del corpo eguali a quelle della loro madre e poppano il latte delle sue mammelle.

A questo punto è necessario ch'io vi rivolga una domanda: lo sapete, ragazzi, come facevano gli antichi a procurarsi il fuoco? No! Ve lo dirò io.

Nei tempi primitivi, quando non era ancor conosciuto l'uso dei metalli, gli uomini si fabbricavano le armi con pezzi di selce e di legno: e mentre attendevano a ciò, s'accorsero che dalla confricazione violenta di queste due sostanze uscivano delle scintille, le quali, poi, divampavano in fiamme. (Baccini 1882: 31-32)

No cabe duda de las habilidades didácticas de la escritora para transmitir los conocimientos y hacer divertir a los niños. Lo 
“Transfer” XV: 1-2 (2020), pp. 276-294. ISSN: 1886-554

que no se entiende es la poca sensibilidad que han tenido los diferentes sistemas educativos, a lo largo de estos últimos dos siglos, despreciando una labor tan encomiable. Razón por la que es necesario recuperar a esta escritora históricamente silenciada. Pero, este tipo de metodología, no fue exclusiva de Ida Baccini sino que era el modelo en auge en la segunda mitad del siglo XIX, y en la actualidad la conocemos gracias a las obras que tuvieron éxito y se mantuvieron como ejemplos didácticos válidos y transcendieron el paso del tiempo. El autor más conocido es Carlo Collodi con su famoso relato Le avventure di Pinocchio. Storia di un burattino. Otro que ha conseguido llegar a nuestra época es Edmondo De Amicis con su libro Cuore. Ambos escritores compartieron con Ida Baccini la experiencia educativa y la filosofía de la unidad de Italia. Por esta razón no se puede entender por qué sus obras siguen siendo conocidas y utilizadas y la amplia publicación de Ida Baccini haya terminado en el olvido total. La reflexión está en las desigualdades de género que siguen vigentes hasta hoy, y es un hecho lamentable, que jamás quitará valor a la labor educativa de Ida Baccini.

A partir de la segunda mitad del siglo XIX cambió un poco la manera de considerar la infancia. Teniendo en cuenta la nueva filosofía post unitaria, era necesario entrar en la mente de los niños y para conseguirlo se empezaron a escribir libros que estuvieran a su nivel de intereses y los atrajeran con historias e ilustraciones divertidas. Nació así la literatura infantil con una clara finalidad pedagógica y educativa que, por primera vez, se proyectaba desde el punto de vista del niño, ya que la infancia era la fase más importante de la vida de una persona (Mugnaini 2002) y de una buena y acertada formación dependería su futuro y el de toda la nación.

Non di rado le donne scrittrici che nell'Ottocento furono attive nel settore pedagogico sono rimaste nell'ombra, soprattutto quelle provenienti dalla media e piccola borghesia, le cui difficoltà finanziarie spingevano spesso ad affiancare all'attività di scrittrici l'insegnamento attivo nelle scuole". (Argenziano 2016: 270) 
“Transfer” XV: 1-2 (2020), pp. 276-294. ISSN: 1886-554

Sin embargo, Ida Baccini, a pesar de su incesante labor en la escuela italiana y de su experiencia docente, decidió cambiar las aulas por la pluma y dedicarse a la elaboración de manuales, el único camino factible para transmitir su pensamiento educativo ya que la escuela la había defraudado porque no estaba a la altura de sus convicciones.

En el siguiente relato, titulado I metalli, se puede percibir el desarrollo didáctico de un tema aburrido y de escaso interés, generalmente bastante difícil de asimilar y de recordar y con tendencia al olvido. Sin embargo, la maestra y escritora, despliega una historia interesante y divertida con la que sería imposible olvidar la composición y función de los metales y transmitir, así, conocimientos básicos de geología:

Attenti, bambini. Oggi dobbiamo parlare di belle cosine, di cosine che vi piaceranno certamente. Guardate bene quest'anello. Lo vedete? Mi sapreste dire di che cos'è, ossia di che sostanza è composto? - È d'oro. - Bravi! Quest'anello, infatti, è d'oro. Ed è pur d'oro il mio orologio, la crocellina che tengo al collo e la moneta che vi feci vedere giorni sono. Com'è bello l'oro, non è vero? L'oro ha un bel colore giallo, risplendente, un colore che quasi potrebbe agguagliarsi a quello del sole. Quando una bambina ha dei bei capelli biondi, diciamo che gli ha d'oro: e anche quando si vuol significare che il grano è maturo, diciamo che la mèsse è color d'oro. Quest'oro così ammirato è una sostanza preziosa, cari figliuoli. Con essa si fanno molte belle cose; sapreste accennarmene qualcuna? Sicuro. Con l'oro si fanno i gioielli che adornano le signore, come sarebbero diademi, buccole, collane, spilli, orologi, anelli, braccialetti, fibbie, medaglioni, ecc. Con l'oro si fanno oggetti di lusso per la tavole, come saliere, porta ampolle, coppe, posate, fruttiere, vassoi, trionfi: e si fanno candelabri per le chiese, reliquiari, cornici, statuette, angioli e croci: anzi vi dirò che in un paese molto lontano dal nostro, la Grecia, fu scolpita, molti secoli sono, una statua, tutta d'oro massiccio. Ora le statue non si scolpiscono più nell'oro, ma nel marmo: e voi ne avrete vedute chi sa quante a ornamento delle piazze, dei palazzi e delle chiese. Con l'oro si coniano le monete da cinque, da dieci, da venti, da cinquanta e da cento lire. Vi auguro di possederne un giorno molte di queste monete; e sapete perchè? Perchè so che siete buoni, $\mathrm{e}$ 
“Transfer” XV: 1-2 (2020), pp. 276-294. ISSN: 1886-554

godete nel dar qualche centesimo ai poverini che non hanno pane. Che cosa fareste, dunque, se vi ritrovaste a posseder tant'oro? Io credo che qui nel vicinato non ci sarebbero più poveri, non è vero? (Baccini 1882: 36)

Un relato que atrae la atención de niños y de adultos y a través del cual es mucho más fácil conocer los metales.

Otro aspecto importante de la obra, intencionalmente educativa, de Ida Baccini es la conjunción de los roles. Tanto hombres como mujeres trabajan juntos para mantener a la familia y para llevar una vida ejemplar. En Il bove se puede apreciar como tanto el padre como la madre contribuyen a la educación de su hijo Attilio, a quien intentan hacer entender la escasa importancia de lo que puedan decir los demás y como forjar una personalidad fuerte y segura de sí misma. El ejemplo moral y comportamental está en al centro de la temática desarrollada, lo que resulta demasiado artificial para nuestros días, pero un modelo didáctico efectivo, sin lugar a dudas.

Un giorno però, il nostro Attilio tornò a casa piangendo e singhiozzando: un bambino cattivo, uno di quei bambini maleducati che vanno alle scuole senza ricavarne profitto, gli aveva dato del bue. Quella parola di bue proferita ad alta voce, con modo schernevole, aveva fatto un grande effetto sull'animo di Attilio: gli pareva di non potere esser trattato di peggio, anche se fosse campato ent'anni.

- Bue! bue! Ma io non ci vedo poi un gran male in questa parola, disse il babbo ridendo. È il nome d'una bestia rispettabile e utilissima, della quale non so come potremmo fare a meno. Attilio spalancava i suoi begli occhi turchini e guardava il babbo con quell'aria che equivale ad una interrogazione (Baccini 1882: 10).

De nuevo Ida Baccini nos enseña que cualquier pretexto es bueno para educar y que ambos padres tienen que estar implicados en la educación, enseñando, de igual manera, a desarrollar la sensibilidad y el respeto por los animales y la naturaleza, como es el caso del relato Gli uccelli: 
“Transfer” XV: 1-2 (2020), pp. 276-294. ISSN: 1886-554

\begin{abstract}
Stamattina una mia cara amica ha voluto regalarmi questo grazioso uccelletto. Guardatelo: è un canarino. Ha le penne gialle, il becco e le gambettine color di rosa pallido; ha gli occhi neri, vispi, brillanti come due margherite. Ora non canta, perchè non ne ha voglia o forse perchè la vista di luoghi nuovi e di persone sconosciute lo intimidisce. Ma quando si sarà addomesticato con noi, allora sentirete. Altro che trilli e gorgheggi di un cantante! Vi parrà impossibile che da un corpicino così piccolo possa uscire tant'onda di melodia. Ma già chi di voi non ha in casa qualche uccellino? Aldo ha un fringuello, Giovanni un cardellino, Tommaso una cutrettola e Gigino un usignuolo. Le care bestioline! (Baccini 1882: 39)
\end{abstract}

Pero lo que más atrae del relato es el uso de figuras literarias que tienen por finalidad desarrollar tanto la imaginación como la sensibilidad de los niños, acercándolos así a la vida familiar

chi di voi non vorrebbe, per un giorno solo, diventare un uccelletto, sol per volare nel luogo o presso la persona che vi è più cara? Quanti bambini volerebbero dalla loro mamma lontana, o al paese dove sono nati e cresciuti e dove riposano in pace le ossa dei loro nonni! (Baccini 1882: 39)

Ida Baccini representa el inicio de una revolución educativa que aún no termina de llevarse a cabo en Italia, una nación, todavía hoy, sumergida en las controversias de la convencionalidad y bajo los dictámenes de la iglesia, tradicional y poco innovadora. Ella, la maestra y la escritora, no solo fue desterrada de los programas escolares sino que se silenció su nombre en el ámbito intelectual y cultural y se despojaron las aulas de sus relatos, lecturas que una vez tuvieron éxito e hicieron mella en tantas consciencias dormidas, y que hoy, podrían despertar los deseos de seguir frecuentando la escuela para aprender, con ilusión y entusiasmo, ahora, en la edad de la imagen y de las nuevas tecnologías en que cada uno de esos personajes podrían cobrar vida, color y movimiento y enseñarnos, cantando, bailando y emocionándonos. "si può infatti qualificare Ida Baccini come figura 'di frontiera', 
“Transfer” XV: 1-2 (2020), pp. 276-294. ISSN: 1886-554

costretta a muoversi all'interno di percorsi identitari codificati ma allo stesso tempo cosciente della necessità di svecchiamento delle figure tradizionali" (Muratore 2006: 7).

\section{Referencias bibliográficas}

ARGENZIANO, Rosa. (2016). "Così parla la buona giovinetta: questioni di morale e di lingua in Come vorrei una fanciulla di Ida Baccini." Italiano LinguaDue, 1: 270-295.

BACCINI, Ida. (1882). Lezioni e racconti per i bambini. Milán: Enrico Trevisini, Editore-Libraio.

---. (1904). La mia vita. Ricordi autobiografici. Società Editrice Dante Alighieri: Roma-Milán.

---. (1918). Memorie di un pulcino e come andò a finire il pulcino. Florencia: Bomporad \& figli.

BARTOLOTTA, Salvatore; GIORDANO PAREDES, María Angélica. (2018). "Ida Baccini: la voz de la infancia". En: CAGNOLATI, Antonella (Coord.): Escritoras en lengua italiana. Renovación del canon literario, Granada: Editorial Comares, pp. 1726.

CANTATORE, Lorenzo. (2014). "Un'identità femminile moderna". L'autobiografia di Ida Baccini. Espacio, Tiempo y Educación $1(1): 31-54$.

CINI, Teresa. (2013). "Ida Baccini, scrittrice per l'infanzia...in molte forme". En Bacchetti, Flavia (ed.). Percorsi della letteratura per l'infanzia. Tra leggere e interpretare. Bolonia: CLUEB Cooperativa Libraria Universitaria Editrice Bolonia, pp. 107116.

COVATO, Carmela. (2006). "Un ambiguo malanno. Diversità, genere e storiografia dell'educazione". En: SANTARONE, Donatello (ed.). Educare diversamente. Migrazioni, differenze, intercultura. Roma: Armando Editore, pp. 231-250.

D'AGOSTINO, Domenico. (2009). "Scrittura ed emancipazione femminile nell'Ottocento italiano". En: GONZÁLEZ DE SANDE, Estela \& CRUZADO RODRÍGUEZ, Ángeles (eds.). Las revo- 
“Transfer” XV: 1-2 (2020), pp. 276-294. ISSN: 1886-554

lucionarias. Literatura e insumisión femenina, Sevilla: ArCiBel Editores, pp. 183-194.

FRAU, Ombretta \& GRAGNANI, Cristina. (2011). Sottoboschi letterari. Florencia: Firenze University Press.

MOSCHINI, Laura. (2013). "L'eterna fanciulla”. En: BRIGANTI, Maria Camilla. (ed.). Autrici per le bambine nell'Ottocento italiano e nella pubblicistica educativa. Roma: Aracne Editrice S.r.l., pp. 13-16.

MUGNAINI, Silvia. (2002). Il libro di testo nell'Italia post-unitaria. Högskolan Dalarna. Akademin Humaniora och Medier. Italienska avdelningen.

MURATORE, Simona. (2006). Pulcini e galline: il pensiero di ida baccini tra pedagogia e femminismo. University of North Carolina: Chapel Hill.

SANTARONE, Donatello. (2006). Educare diversamente. Migrazioni, differenze, intercultura. Roma: Armando Editori.

SCOLARI SELLERIO, Arianna. (1963). "Ida Baccini" Enciclopedia Treccani. Dizionario Biografico degli Italiani, 5: 135-138.

SOLDANI, Simonetta. (2004). "Donne educanti, donne da educare. Un profilo della stampa femminile toscana (1770-1945)". En: FRANCHINI, Silvia \& SOLDANI, Simonetta. (ed.). Donne e giornalismo. Percorsi e presenze di una storia di genere. Milán: FrancoAngeli, pp. 309-362.

TORTORELLI, Gianfranco. (2006). Il torchio e le torri. Editoria e cultura a Bologna dall'unitá al secondo dopoguerra. Bolonia: Pendragon.

Fecha de recepción: 2.09. 2019 Fecha de aceptación: 01.10.2019

\section{Resumen:}

La obra y la personalidad de Ida Baccini se funden en un universo variopinto de ideas, instrucción y reclamos innovadores, en un marco sociopolítico liderado por cambios e importantes reformas. En ese momento de transición histórica, la nueva Italia unida, necesitaba un modelo de sociedad cuya ejemplaridad creara los 
“Transfer” XV: 1-2 (2020), pp. 276-294. ISSN: 1886-554

pilares de una nación fuerte e instruida. A esa llamada acude la maestra, escritora, periodista e inquieta idealista para marcar los inicios de una buena formación académica y dejar su huella, totalmente innovadora, en los manuales escolares y en sus cuentos infantiles y juveniles que contribuirían enormemente al desarrollo de la educación italiana; aunque, lamentablemente, se haya quedado en el olvido y sus obras no tengan eco más allá de las fronteras. A este rescate contribuiremos en el presente artículo.

Palabras clave: Educación, Innovación, Manuales, Cuentos infantiles, Traducción.

\title{
IDA BACCINI'S EDUCATIONAL UNIVERSE: PASSION, COMMITMENT AND PERSONALITY
}

\begin{abstract}
:
The work and personality of Ida Baccini merge into a diverse universe of ideas, instruction and innovative claims, within a socio-political framework led by changes and important reforms. In that moment of historical transition, the new united Italy needed a model of society whose exemplarity created the pillars of a strong and educated nation. To this call comes the teacher, writer, journalist and restless idealist to mark the beginnings of a good academic education and leave their mark, totally innovative, in school textbooks and their children's and young stories that would greatly contribute to the development of Italian education; although, unfortunately, it has been forgotten and its works have no echo beyond the borders. To this rescue we will contribute in the present article.
\end{abstract}

Keywords: Education, Innovation, Manuals, Children's stories, Translation. 